\title{
A 36 nucleotide deletion mutation in the coding region of the NS1 gene of an influenza $A$ virus RNA segment 8 specifies a temperature-dependent host range phenotype
}

\author{
Mark H. Snyder ${ }^{1}$, William T. London ${ }^{2}$, Hunein F. Maassab ${ }^{3}$, \\ Robert M. Chanock ${ }^{1}$ and Brian R. Murphy ${ }^{1}$ \\ ${ }^{1}$ Laboratory of Infectious Diseases, NIAID, ${ }^{2}$ Infectious Diseases Branch, NINDS, National Institutes of \\ Health, Bethesda, MD 20892, U.S.A. and ${ }^{3}$ University of Michigan School of Public Health, Ann Arbor, MI \\ 48104, U.S.A.
}

(Accepted 6 October 1989)

\section{Summary}

Previously a spontaneous 36 nucleotide deletion in the coding region of NS1 was detected in the NS gene of a reassortant virus (CR43-3) recovered from a dual infection by the influenza A/Ann Arbor/6/60 cold-adapted ( $c a$ ) mutant and wild-type (wt) influenza A/Alaska/6/77 (H3N2). The hemagglutinin, neuraminidase and NS genes were derived from the wild type virus parent while the other 5 genes were derived from the $c a$ parent. The CR43-3 reassortant virus exhibited: (i) a host range ( $h r)$ phenotype, i.e. the reassortant replicated efficiently in avian cells in tissue culture but failed to grow in mammalian (MDCK) cell culture and (ii) an attenuation (att) phenotype, i.e., the reassortant was restricted in replication in the upper and lower respiratory tract of ferrets and hamsters. Since the CR43-3 reassortant possessed 5 genes from the $c a$ parent which are each known to contain one or more mutations, it was not possible to assign the $h r$ and att phenotypes solely to the NS deletion mutant gene. In order to determine the phenotype(s) specified solely by the mutant NS gene, it was transferred into a reassortant virus (143-1) which derived its seven other genes from the homologous wild type A/Alaska/6/77 virus. The deletion mutant NS gene specified only a partial $h r$ phenotype manifested by a reduction in plaque size in MDCK tissue, but not a

Correspondence to: Dr Brian R. Murphy, Building 7, Room 100, LID, NIAID, Bethesda, MD 20892, U.S.A. 
reduction in plaque number. Thus, the complete $h r$ manifested by the CR43-3 parent virus is specified by the mutant NS1 gene acting in concert with one or more genes derived from the $c a$ virus. The clone 143-1 virus exhibited the $t s$ phenotype and was restricted in plaque formation at $37^{\circ} \mathrm{C}$ in $\mathrm{MDCK}$ cells, a level of temperature sensitivity previously shown with other $t s$ mutants to correlate with significant restriction of viral replication in the lower respiratory tract of hamsters. However, the clone 143-1 virus grew almost as well as the wt virus in the upper and lower respiratory tracts of hamsters and chimpanzees and thus did not possess the att phenotype. The finding that the $t s$ phenotype was not manifest in vivo in animals with a $37^{\circ} \mathrm{C}$ core temperature indicates that the mutated NS1 gene specifies a host dependent $t s$ phenotype with replication restricted in vitro (MDCK tissue culture) at $37^{\circ} \mathrm{C}$ but not in vivo in the lungs of hamsters and chimpanzees. $t s^{+}$virus was readily recovered from infected hamsters and chimpanzees indicating that the $t s$ phenotype specified by the 36-base deletion was not stable following replication in vivo. This particular NS mutant gene would not be a suitable attenuating gene for inclusion in a live virus vaccine.

Influenza virus; Deletion mutation; Host-dependent $t s$ phenotype

\section{Introduction}

Numerous approaches to attenuate viruses for use as live virus vaccines have been developed (Chanock et al., 1988). Among these, deletion of an appreciable region of the virus genome has a theoretical appeal because this form of mutation cannot be corrected by spontaneous restoration of the deleted sequence. Thus, attenuation specified by a deletion mutation might be more stable than that specified by a single point mutation or a limited number of point mutations. This approach has remained theoretical for influenza $A$ virus because techniques are not currently available to produce specific deletions in the genome of negative-stranded RNA viruses either directly or through the transfer of genetically engineered cloned DNA back into the viral genome. Thus, an analysis of the effect of deletion mutation on virulence of influenza A virus depends on the identification of spontaneous deletion mutations. Recently, the isolation of the influenza A/Alaska/ 6/77 (H3N2) x A/Ann Arbor/6/60 (H2N2) cold-adapted (ca) CR43-3 reassortant virus which spontaneously sustained a 36 base deletion in the region of RNA segment 8 which encodes the nonstructural protein NS1 provided an opportunity to test the level of attenuation and phenotypic stability of an influenza A virus containing a deletion mutation of moderate size (Maassab and DeBorde, 1983). Analysis of the nucleotide sequence of the mutant NS gene demonstrated that it was identical to that of the A/Alaska/ 77 wild type virus except for the 36 base deletion (amino acids 66-77 of NS1 protein) (Buonagurio et al., 1984). Analysis of the genotype of reassortant virus revealed that the genes which encoded the hemagglutinin and neuraminidase were derived from the influenza A/Alaska/ 77 (H3N2) 
wt virus parent whereas the five non-surface glycoprotein genes other than the mutated segment were derived from the A/Ann Arbor/6/60 $\mathrm{ca}$ donor virus.

Clone CR43-3 virus exhibited a host-dependent restriction of replication ( $h r$ phenotype) not exhibited by either parent. It replicated efficiently in eggs and formed plaques on primary chick kidney (PCK) tissue culture, but failed to produce plaques on Madin-Darby canine kidney (MDCK) tissue culture (Maassab and DeBorde, 1983). Previous genetic analysis suggested that the $h r$ phenotype cosegregated with the deleted NS gene (Maassab and DeBorde, 1983). However, this interpretation was not definitive because each reassortant bearing the deleted NS gene also contained one or more genes derived from the mutant A/AA/6/60 ca donor virus (Maassab and DeBorde, 1983). Furthermore, the CR43-3 virus possessed two properties of the A/AA/6/60 $\mathrm{ca}$ parent virus, attenuation (att) in ferrets and temperature sensitivity ( $t s$ ) of replication in PCK tissue. Interestingly, attenuation in ferrets involved restriction in replication in both the upper and lower respiratory tract, a property not shared by either parent virus. However, the relative contribution of the deletion mutant NS1 gene to the full spectrum of phenotypes exhibited by the CR43-3 virus was not clear.

Several properties of the CR43-3 reassortant would be useful in a live virus vaccine strain, namely efficient replication in embryonated chicken eggs (which is an acceptable substrate for vaccine production) and restriction of replication in animals specified by a genetically stable deletion mutation. To define the phenotype(s) specified by the NS1 deletion mutation, we produced a reassortant virus, clone 143-1, which contained the mutant NS1 gene in a background of genes from the homologous A/Alaska/ 77 wt virus from which it was derived. The recombinant was evaluated for the $h r$ and $t s$ phenotypes in vitro, and the att phenotypes in hamsters and chimpanzees. The findings indicated that the deletion mutation specified: (1) a $t s$ phenotype independent of that specified by A/AA/6/60 ca genes (Snyder et al., 1988); (2) a partial $h r$ phenotype; and (3) little if any restriction of replication in vivo. Furthermore, the $t s$ phenotype specified by the deletion mutation was not stable after replication in vivo. The implications of these findings for vaccine development are discussed.

\section{Materials and Methods}

\section{Viruses}

The isolation and cloning of the influenza A/Alaska/6/77 (H3N2) wt virus (Murphy et al., 1980) and the reassortant A/Alaska/6/77 x A/Ann Arbor/6/60 (H2N2) ca reassortant CR43-3 virus were described previously (Maassab and DeBorde, 1983). The isolation and evaluation of the reassortant clone $t s-55$ derived from the mating of influenza A/Udorn/307/72 (H3N2) $t s-368 \times \mathrm{A} /$ Victoria/3/75 (H3N2) $t s-65$ reassortant were reported previously (Murphy et al., 1982a). The isolation, cloning, and characterization of $\mathrm{A} /$ Bethesda/1/85 (H3N2) wt virus is 
described elsewhere (Sears et al., 1988). Each virus was subjected to one additional passage in 9-day old embryonated eggs.

A single gene reassortant virus containing the partially deleted NS1 gene was produced by coinfecting PCK tissue culture at a multiplicity of infection of one with the A/Alaska/6/77 virus and the CR43-3 reassortant virus (Snyder et al., 1987). The coinfected cultures were incubated at $33^{\circ} \mathrm{C}$ and harvested $24 \mathrm{~h}$ later. Plaque progeny were picked from tissue culture monolayers and amplified in embryonated eggs as previously reported (Snyder et al., 1987) and their genotype was determined as described below. The single gene reassortant virus, clone 143-1, that contained the partially deleted NS1 gene in a background of A/Alaska/ 77 wt virus genes was then biologically cloned by plaque-to-plaque passage on PCK tissue culture monolayers and amplified in embryonated eggs. This virus, clone 143-1, was mated in a similar manner with an $\mathrm{A} /$ Bethesda/ $1 / 85$ wild type virus as above and plaque progeny were evaluated for their $t s$ and $h r$ phenotype, and the genotype of each progeny virus was determined.

\section{Genotype of reassortant viruses}

The parental origin of the RNA segments of each reassortant virus was determined following polyacrylamide gel electrophoresis by comparison of their migration with those of the corresponding parental genes. Virus was purified and RNA extracted as previously described (Tian et al., 1985). Purified RNA was analyzed in polyacrylamide gels containing $2.6 \%$ acrylamide and either $6.0 \mathrm{M}$ or 7.0 M urea as described previously (Murphy et al., 1984). RNA segments were visualized by ammoniacal silver staining (Boulikas and Hancock, 1981).

\section{Efficiency of plaque formation (EOP) at permissive and restrictive temperatures}

The EOP of each virus was determined in PCK or MDCK tissue cultures at 37, 38 , and $39^{\circ} \mathrm{C}$ and compared with that at $33^{\circ} \mathrm{C}$ as described (Murphy et al., 1982b).

\section{Studies in hamsters}

Evaluation of virus replication in four-week old female Golden Syrian hamsters was performed as described previously (Murphy et al., 1982a). Hamsters were anesthesized and inoculated intranasally with $10^{5}$ tissue culture infectious dose $\left(\mathrm{TCID}_{50}\right)$ of wt or reassortant virus in a $0.1 \mathrm{ml}$ suspension. Five hamsters in each group were sacrificed on each of days 1-4 and $10 \% \mathrm{w} / \mathrm{v}$ suspensions of the nasal turbinates and lungs of each hamster were prepared. The virus titers were determined in MDCK or PCK tissue culture and mean titers were expressed as $\mathrm{TCID}_{50} / \mathrm{g}$ of tissue.

In an additional study, hamsters were inoculated intranasally with $10^{5} \mathrm{TCID}_{50}$ of a reassortant virus in an $0.1 \mathrm{ml}$ inoculum. To prolong virus replications, hamsters received cyclophosphamide (Mead Johnson and Co., Evansville, IN) intraperitoneally according to the following schedule: day $2,150 \mathrm{mg} / \mathrm{kg}$; days $5,8,11$, and 14 , 
$100 \mathrm{mg} / \mathrm{kg}$. Fifteen hamsters from each group were sacrificed on days $5,7,9,12$, and 15. Virus isolates were obtained in MDCK tissue culture as described above and the EOP of the virus isolates was characterized at $33^{\circ} \mathrm{C}$ and $38^{\circ} \mathrm{C}$ on MDCK monolayers.

\section{Studies in chimpanzees}

Studies in chimpanzees were performed as described (Snyder et al., 1986). Briefly, young chimpanzees were anesthesized and inoculated intranasally and intratracheally with a $0.5 \mathrm{ml}$ inoculum which contained $10^{7} \mathrm{TCID}_{50}$ of virus. Virus isolation and quantitation was performed on nasopharyngeal swab specimens obtained daily for 10 days post-inoculation and on tracheal lavage specimens obtained on days 2, 4,6 , and 8 post-inoculation. The EOP of the virus isolates was characterized at $33^{\circ} \mathrm{C}$ and $38^{\circ} \mathrm{C}$ on MDCK monolayers.

\section{Results}

\section{Characterization of reassortant clone 143-1}

Reassortant virus 143 , isolated from the mating of $\mathrm{A} /$ Alaska/6/77 (H3N2) wt $\mathrm{x}$ CR43-3, derived the NS gene carrying the deletion mutation from CR43-3 while its seven other genes came from wt virus (Fig. 1). The reassortant clone 143-1 virus formed plaques in both MDCK and PCK tissue culture, whereas CR43-3 formed plaques only on avian tissue culture (Table 1). However the plaques formed by clone 143-1 on MDCK cells were small (1-2 $\mathrm{mm}$ in diameter) compared with those produced by the A/Alaska/77 wt virus (approximately $4-6 \mathrm{~mm}$ ). This indicates that the NS deletion specified only a partial $h r$ phenotype in which plaque formation in mammalian cells did not appear to be restricted but plaque size was reduced.

The level of temperature sensitivity of the parent CR43-3 and its single gene reassortant virus, clone 143-1 in PCK tissue, was similar indicating that the mutant NS1 gene specified the $t s$ phenotype. Furthermore, the degree of temperature sensitivity manifested by clone 143-1 virus in MDCK cells was greater than in PCK cells suggesting that the deletion mutation specifies a host-dependent $t s$ phenotype in which the EOP of the virus at restrictive temperature is host cell-dependent. To confirm that the $t s$ phenotype present in the clone 143-1 reassortant virus was indeed specified by the mutated NS1 gene and not the fortuitous result of a spontaneous $t s$ mutation in another gene, segregational analysis was undertaken by mating the clone 143-1 with another wt (A/Bethesda/1/85) virus and by determining the genotype and the $t s$ phenotype of the virus progeny (Table 2). The $t s$ phenotype cosegregated with the mutant NS1 gene. The small plaque phenotype also cosegregated with the mutated NS gene further supporting our finding that the deletion mutation specifies a partial $h r$ phenotype. These results suggested that the 


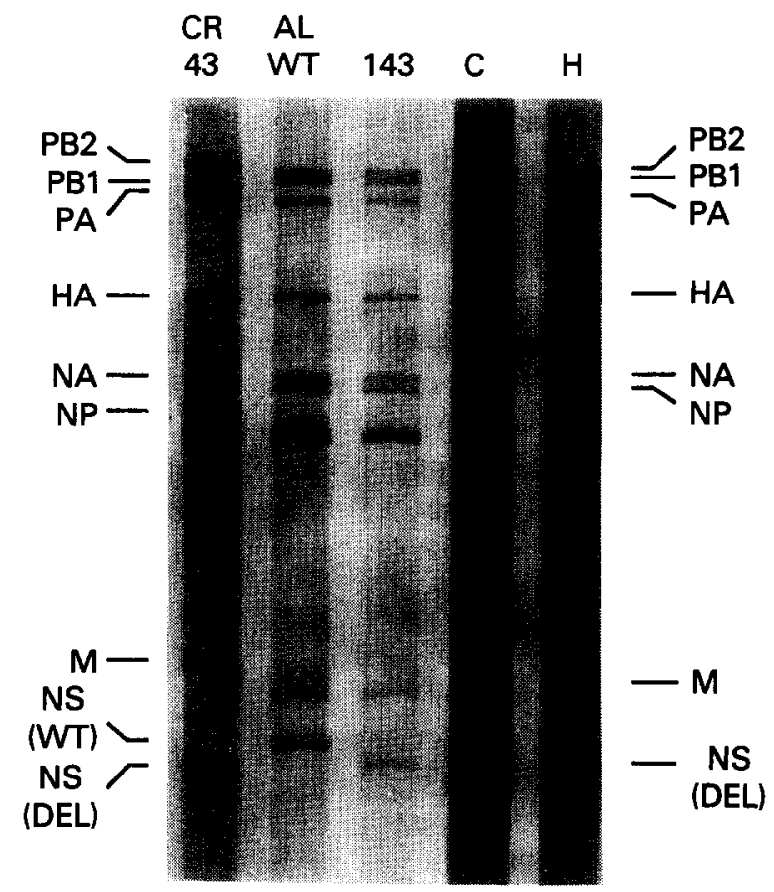

Fig. 1. The genotype of $t s$ clone 143-1 and the $t s^{+}$virus recovered from hamsters $(\mathrm{H})$ and chimpanzees (C) is compared to that of its CR-43 and A/Alaska/77 wild-type parents. Each $t s^{+}$was recovered from a single plaque. The polyacrylamide gels contained $2.6 \%$ acrylamide and $6.0 \mathrm{M}$ urea and were electrophoresed at $90 \mathrm{~V}$ at $0.5^{\circ} \mathrm{C}$ for $16 \mathrm{~h}$.

expression of the complete $h r$ phenotype, i.e., failure to produce plaques in MDCK cells, requires cooperation of the deletion mutant NS1 gene and one or more genes of the $c a$ parent.

\section{TABLE 1}

Genotype and $t s$ and $h r$ phenotypes of influenza A/Alaska/77 (H3N2) wild-type or reassortant viruses

\begin{tabular}{lllllllll}
\hline Virus & \multicolumn{1}{l}{ Genotype } & & & & & \\
\cline { 2 - 8 } & PB2 & PB1 & PA & HA & NA & NP & M & NS \\
\hline CR43-3 & C & C & C & A & A & C & C & A(Del) \\
143-1 & A & A & A & A & A & A & A & A(Del) \\
Wild-type & A & A & A & A & A & A & A & A \\
\hline
\end{tabular}

${ }^{a} \mathrm{C}=$ RNA segment derived from influenza A/Ann Arbor/6/60 (H2N2) ca virus. A = RNA segment derived from influenza A/Alaska/6/77 (H3N2) wild-type virus. A(Del) = RNA segment 8 originally derived from the influenza A/Alaska/77 wild-type virus which sustained a 36 base deletion in the NS1 coding region.

b Shut-off temperature is defined as the lowest temperature at which a 100 -fold reduction of virus titer is seen compared to that at $33^{\circ} \mathrm{C}$. 


\section{Virus replication in hamsters}

Our previous studies indicated that viruses that have a $37^{\circ} \mathrm{C}$ shut-off temperature of plaque formation were markedly restricted in replication in the lower respiratory tract of the hamster, an animal with a $37^{\circ} \mathrm{C}$ core body temperature (Murphy et al., 1976, 1982a). For this reason we expected that clone 143-1 virus would be markedly restricted in replication in the lungs of hamsters. We also expected that the isolates obtained from infected hamsters would retain their $t s$ phenotype after replication in vivo because the $t s$ phenotype was specified by a deletion mutation. We compared the replication in hamsters of CR43-3 and clone 143-1 viruses with that of $\mathrm{A} /$ Alaska/ 77 wt virus. We failed to recover parental CR43-3 virus from the upper or lower respiratory tract of hamsters after intranasal inoculation (Table 3). In contrast, reassortant progeny clone 143-1 replicated in both the upper and lower respiratory tract of the hamsters, and the peak titers attained in the upper and lower respiratory tract of the hamster were comparable to those of the A/Alaska/ 77 wt virus. The observed efficient replication of virus in the lower respiratory tract is in contrast to our experience with each of the other $37^{\circ} \mathrm{C}$ ts viruses which were studied (Murphy et al., 1982a; Murphy et al., 1976). This suggested that the ts phenotype was a manifestation of a host-dependent $t s$ (hd- $t s$ ) mutation that permitted efficient virus replication in the lungs $\left(37^{\circ}\right)$ of hamsters but restricted virus replication in MDCK cells incubated at $37^{\circ} \mathrm{C}$. However, clone 143-1 virus was cleared from the lower respiratory tract earlier than the wild-type virus and was recovered in slightly lower titers, suggesting that there might have becn a slight influence of the deletion mutation on virus replication in animals. Also, clone 143-1 virus was recovered from the lungs of only one of five hamsters on day 3 p.i. In contrast the A/Alaska/ $77 \mathrm{wt}$ virus was isolated from the lungs of each of four hamsters sacrificed on day 4 p.i.

Six isolates of clone 143-1 obtained from the lungs of infected hamsters on days one and two and fifteen isolates obtained from the nasal turbinates on days one through four p.i. were $t s$ in MDCK tissue culture. This indicates that the high titer of replication of clone 143-1 virus was not due to the emergence of $t s^{+}$virus and that the $t s$ phenotype was stable after the normal course of virus replication in hamsters.

\begin{tabular}{|c|c|c|c|c|c|c|c|c|}
\hline \multicolumn{6}{|c|}{ Virus titer $\left(\log _{10} \mathrm{pfu} / \mathrm{ml}\right)$ in indicated tissue } & \multicolumn{2}{|c|}{ Shut-off ${ }^{b}$ temperature } & \multirow{3}{*}{$\begin{array}{l}h r^{c} \\
\text { phenotype }\end{array}$} \\
\hline \multicolumn{3}{|l|}{$\overline{\mathrm{PCK}}$} & \multicolumn{3}{|c|}{ MDCK } & \multirow[t]{2}{*}{ PCK } & \multirow[t]{2}{*}{ MDCK } & \\
\hline $33^{\circ} \mathrm{C}$ & $37^{\circ} \mathrm{C}$ & $38^{\circ} \mathrm{C}$ & $33^{\circ} \mathrm{C}$ & $37^{\circ} \mathrm{C}$ & $38^{\circ} \mathrm{C}$ & & & \\
\hline 6.3 & 5.0 & $\leq 3.0$ & $\leq 3.0$ & $\leq 3.0$ & $\leq 3.0$ & $38^{\circ} \mathrm{C}$ & NA & complete \\
\hline 5.9 & 4.5 & 3.0 & 6.2 & $\leq 2.0$ & $\leq 2.0$ & $38^{\circ} \mathrm{C}$ & $37^{\circ} \mathrm{C}$ & partial \\
\hline 6.3 & 6.9 & 5.3 & 7.5 & 7.3 & 7.5 & $\geq 39^{\circ} \mathrm{C}^{\mathrm{d}}$ & $\geq 39^{\circ} \mathrm{C}^{\mathrm{d}}$ & absent \\
\hline
\end{tabular}

c Complete $h r$ phenotype is defined as the ability to produce plaques on monolayer cultures of PCK at permissive temperature $\left(33^{\circ} \mathrm{C}\right)$ but not on cultures of MDCK. Partial $h r$ phenotype is indicated by the ability to form plaque on MDCK cells but the plaque size was smaller than that of wild-type virus.

d Data not shown. 


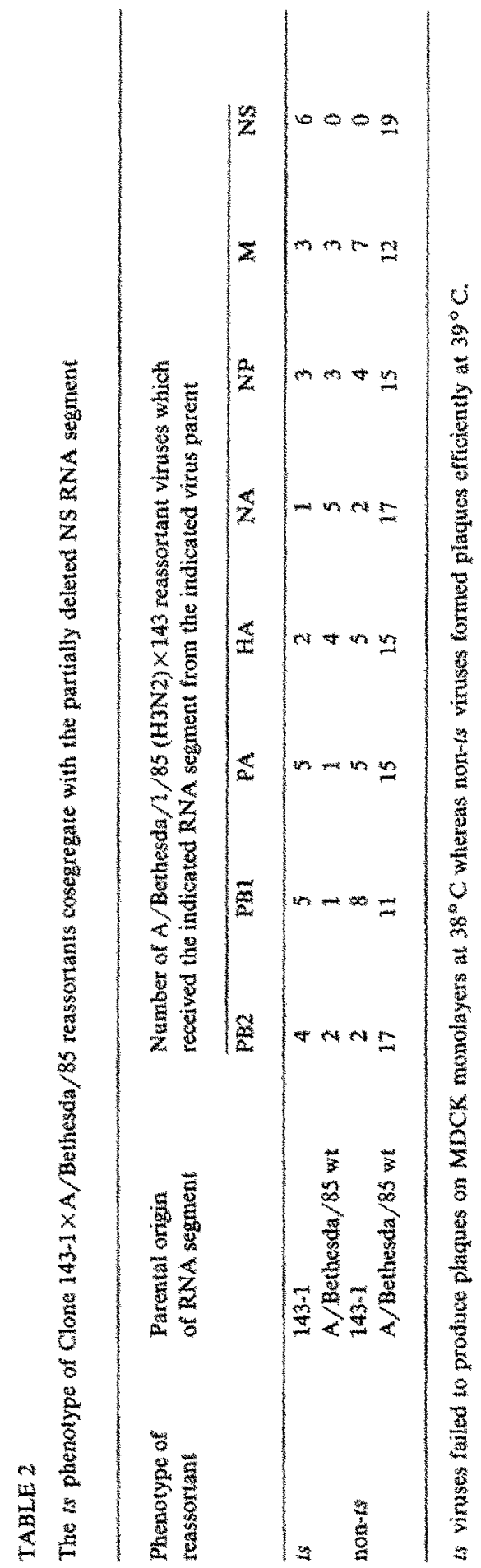


TABLE 3

Replication of $\mathrm{A} / \mathrm{Alaska} / 77$ (H3N2) wild-type or reassortant viruses in hamsters

\begin{tabular}{|c|c|c|c|c|}
\hline \multirow{3}{*}{$\begin{array}{l}\text { Virus } \\
\text { administered }\end{array}$} & \multicolumn{4}{|c|}{ Day of peak titer (mean $\log _{10} \mathrm{TCID}_{50} / \mathrm{g}$ ) ${ }^{\mathrm{a}}$ in indicated tissue } \\
\hline & \multicolumn{2}{|c|}{ Nasal turbinates } & \multicolumn{2}{|c|}{ Lungs } \\
\hline & Day & $\begin{array}{l}\text { Peak } \\
\text { titer }\end{array}$ & Day & $\begin{array}{l}\text { Peak } \\
\text { titer }\end{array}$ \\
\hline CR43-3 & - & $\leq 1.5$ & - & $\leq 1.5$ \\
\hline 143-1 & 3 & $4.7 \pm 0.2$ & 2 & $4.7 \pm 0.3$ \\
\hline Wild-type & 2 & $5.2 \pm 0.4$ & 2 & $5.7 \pm 0.7$ \\
\hline
\end{tabular}

Hamsters were inoculated intranasally with $10^{5} \mathrm{TCID}_{50}$ of virus. On each of days $1-4$ post-inoculation five hamsters in each group were sacrificed and a $10 \%$ (wt/vol) suspension of their nasal turbinates and lungs were prepared. The lowest detectable quantity of virus was $10^{1.5} \mathrm{TCID}_{50} / \mathrm{g}$. For each of the values reported the means of the five animals are presented. Virus was not recovered from the lungs or nasal turbinates of animals inoculated with CR43-3.

a \pm standard error.

\section{Virus replication in chimpanzees}

Attenuation of influenza A viruses for rodents is not always associated with attenuation for primates, including humans (McCahon and Schild, 1972). In order to evaluate more fully the usefulness of the NS1 deletion mutation for the production of attenuated reassortant virus for use as live virus vaccines in humans, we compared the replication of virus clone 143-1 in chimpanzees with that of the A/Alaska/ 77 wt virus. We chose chimpanzees because the normal body temperature of the chimpanzee and humans is the same. Again, we found that the NS1 deletion mutation did not significantly restrict virus replication in either the upper or lower respiratory tract (Table 4). Furthermore, virus isolated as early as two days after inoculation contained $t s^{+}$phenotypic revertants that formed plaques on MDCK monolayers at $38^{\circ} \mathrm{C}$. Of interest was the observation that $t s^{+}$virus was replaced by $t s$ virus later in the course of infection of each chimpanzee. Genotype analysis demonstrated that RNA segment 8 of the $t s^{+}$revertant virus comigrated with that of the clone 143-1 reassortant virus administered indicating that the NS1 deletion mutation was still present in the $t s^{+}$phenotypic revertant virus (Fig. 1).

\section{Stability of the ts phenotype after virus replication in immunosuppressed hamsters}

Following the detection of instability of the $t s$ phenotype specified by the NS1 deletion, the frequency of phenotypic reversion of the reassortant bearing the NS mutant gene was compared to that of a virus whose $t s$ defect was thought to be specified by missense mutations. Previously, we determined the level of phenotypic stability of an influenza A ts virus, $t s-55$ virus, which contains two genes (PB2 and NP) with $t s$ mutations, after prolonged replication in immunosuppressed animals and found it to be our most stable $t s$ virus (Murphy et al., 1982a). Therefore, we compared the stability of the clone 143-1 virus with that of the $t s-55$ in immunosup- 


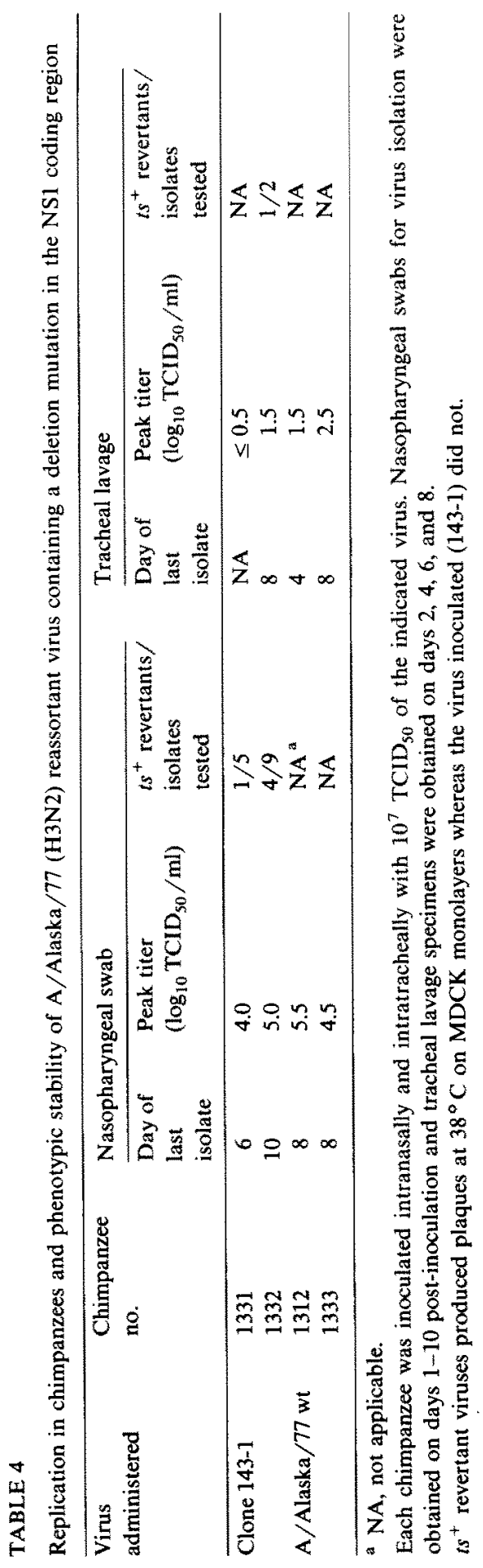


TABLE 5

The stability of the $t s$ phenotype of clone 143-1 virus after replication in immunosuppressed hamsters

\begin{tabular}{|c|c|c|c|c|}
\hline \multirow{2}{*}{$\begin{array}{l}\text { Virus } \\
\text { administered }\end{array}$} & \multicolumn{3}{|c|}{ Number of isolates with $t s^{+}$virus/number of isolates tested } & \\
\hline & Days 5-9 & Days $12-15$ & Total & \\
\hline $143-1$ & $5 / 17$ & $7 / 14$ & $\left.\begin{array}{r}12 / 31 \\
4 / 37\end{array}\right]$ & $P<0.05^{a}$ \\
\hline
\end{tabular}

${ }^{a}$ Fisher's exact test (two-tailed).

Hamsters received $10^{5} \mathrm{TCID}_{50}$ of virus intranasally and were immunosuppressed by injection of cyclophosphamide. Hamsters were sacrificed on days $5,7,9,12$, and 15 post infection and the EOP of virus isolated from the nasal turbinates was tested at $33^{\circ} \mathrm{C}$ and $38^{\circ} \mathrm{C}$ and was then compared to that of the parental virus. Isolates with virus that produced plaques at $38^{\circ} \mathrm{C}$ were considered $t s^{+}$. Virus was not recovered from the lungs of these animals.

pressed hamsters. Hamsters were inoculated with either clone 143-1 or the previously described $t s-55$ virus and then immunosuppressed by the administration of cyclophosphamide in order to allow virus replication to continue for up to 15 days. Virus isolates were obtained from the nasal turbinates of infected hamsters and characterized for their expression of the $t s$ phenotype. Unexpectedly, we failed to isolate 143-1 virus from the lungs of infected animals which suggested that the immunosuppressed animals cleared the virus from their pulmonary tissue by day 5 . The frequency of isolation of $t s^{+}$revertants from the nasal turbinates of hamsters infected with clone 143-1 was significantly greater than that of $t s-55$ (Table 5). The 8th RNA segment of the $t s^{+}$virus recovered from hamsters co-migrated with that of the 143-1 input virus (Fig. 1).

\section{Discussion}

The phenotypes specified by the 36 base pair deletion mutation in the NS1 coding region of RNA segment 8 of the CR43-3 reassortant virus were defined in the present study. This was accomplished by segregating the mutant NS1 gene, originally derived from the A/Alaska/ 77 wt virus, into a reassortant virus, clone 143-1, containing a background of A/Alaska/ 77 wt virus genes. This reassortant was restricted in plaque formation at $37^{\circ} \mathrm{C}$ indicating that the deletion mutation specified the $t s$ phenotype in MDCK tissue culture. The CR43-3 parent virus was also $t s$, but it possessed the PB1 and PB2 genes from the A/Ann Arbor/6/60 (H2N2) $c a$ donor virus which are known to possess $t s$ mutations (Snyder et al., 1988). It was not until the partially deleted NS1 gene was segregated from the other $t s$ genes derived from the $c a$ donor virus that its $t s$ property became fully apparent. This finding that the mutant gene specified a shut-off temperature for plaque formation of $37^{\circ} \mathrm{C}$ was of interest because this level of temperature sensitivity in vitro was previously shown to satisfactorily attenuate influenza $\mathrm{A}$ virus for susceptible humans (Murphy et al., 1972; Tolpin et al., 1981). The previously studied $t s$ viruses possessing putative missense mutations were phenotypically unstable after 
replication in hamsters and humans (Tolpin et al., 1981). It was anticipated that a virus containing an appreciable deletion in the NS1 gene would be restricted in replication in the lower respiratory tract of animals and that the virus would be phenotypically stable, but these expectations were not borne out by our in vivo studies.

The CR43-3 parent virus possessed an att phenotype not present in either parent virus, i.e., complete absence of replication in the respiratory tract of ferrets. This att phenotype segregated with the RNA segment 3, NP, and mutated NS1 gene (Maassab and DeBorde, 1983). In the present study we demonstrated that the att phenotype of restricted replication in ferrets of the parental CR43-3 reassortants was also manifest in hamsters. However, the clone 143-1 reassortant possessing only the mutant NS1 gene from CR43-3 did not exhibit the att phenotype in hamsters. Thus, the att phenotype was specified by the mutant NS1 gene plus CR43-3 RNA segment 3 or NP gene or both these genes. Unexpectedly, the clone 143-1 virus replicated to high titer (compared to wt virus) in the lower respiratory tract despite its $37^{\circ} \mathrm{C}$ in vitro shut-off temperature. Missense $t s$ mutants with a $37^{\circ} \mathrm{C}$ shut-off temperature are almost completely restricted in replication in the lower respiratory tract of hamsters (Murphy et al., 1982a; Murphy et al., 1976). The virus recovered from hamsters on the peak day of replication of the clone 143-1 reassortant was $t s$ indicating that loss of the $t s$ phenotype was not responsible for the observed high level of replication. The explanation most consistent with these observations is that the deletion mutation in the NS1 gene specifies a temperature-dependent host range $(t d-h r)$ phenotype in which the virus is not restricted in replication in hamsters but is restricted in replication at $37^{\circ} \mathrm{C}$ in MDCK tissue culture. Such $t d-h r$ mutations have been identified in influenza A viruses with putative missense mutations in the NS gene (Shimizu et al., 1983). In those $t d-h r$ mutants the permissive tissue was rhesus monkey kidney tissue culture and the restriction of replication at elevated temperature was manifested in MDCK tissue. However, for the clone 143-1 $t d-h r$ mutants the permissive tissue is the hamster respiratory tract epithelium and the restrictive tissue (at $37^{\circ} \mathrm{C}$ ) is MDCK.

In the present study, we also sought evidence for the att phenotype of clone 143-1 virus in chimpanzees and found that the level of replication in the upper and lower respiratory tract was only slightly less than that of wt. These results indicated that the RNA segment bearing the NS1 deletion has little promise as an attenuating gene for use in a live virus vaccine strain for humans. Interestingly, large deletions in the carboxy terminus of NS1 are not associated with attenuation indicating that various domains of the NS1 gene are not required for efficient replication in vivo (Norton et al., 1987; Parvin et al., 1983).

The last phenotype manifested by the CR43-3 virus was the $h r$ phenotype in which the mutant failed to replicate in MDCK cells at any temperature tested. The $h r$ phenotype, like the att phenotype, cosegregated with a set of genes in the CR43-3 virus, namely RNA3, NP, and the deleted NS1 gene (Maassab and DeBorde, 1983). The clone 143-1 reassortant virus had only a partial $h r$ phenotype manifested by a decrease in plaque size in MDCK tissue but not in plaque number. Thus, the $h r$ and att phenotypes of the CR43-3 virus require a cooperation of the 
partially deleted NS1 gene and the CR43-3 RNA 3 and/or NP genes derived from the A/Ann Arbor/6/60 $c a$ donor virus. Cooperation of influenza virus genes in the establishment of the $h r$ and att phenotypes has been described and discussed previously (Snyder et al., 1987).

It had been assumed that the phenotype specified by a deletion mutation would be more stable than the phenotype specified by missense mutation. This is likely to be the case when genes that are not essential for replication in vitro and in vivo are completely deleted. Thus, a herpes simplex virus (HSV) from which the thymidine kinase (tk) gene has been deleted was more stable in phenotype after replication in vivo than a HSV virus bearing a missense mutation in the tk gene (Meignier et al., 1988; Tenser and Dunstan, 1979). However, this rule is not absolute in that the phenotype of an animal virus bearing a deletion mutation can be modified by a second-site mutation (Barkan et al., 1987; Dildine and Semler, 1989; Preston et al., 1988). In the present study, we demonstrate that the $t s$ phenotype specified by an internal deletion ( 12 of the 245 amino acids deleted) in the NS1 protein can be modified following replication in vivo. We were able to isolate $t s^{+}$virus from chimpanzees and immunosuppressed hamsters. The $t s^{+}$recovered virus has an NS1 gene of the same size as the input virus as judged by gel electrophoresis. The frequency of loss of $t s$ phenotype was greater for the deletion mutant than for a $t s$ missense mutant containing two genes bearing $t s$ mutations. The locus and sequence of the correcting mutation is currently being studied but it should prove to be either an intragenic or extragenic suppressor mutation because the original deletion mutation appears to be present in the phenotypic revertant virus. These findings have implications for vaccine development in those instances in which attenuation is produced by partial deletion of a gene.

\section{Acknowledgements}

We would like to acknowledge the excellent technical assistance of Frank Wood and the excellent editorial assistance of Linda Jordan. This work was supported by Grant N01AI52564.

\section{References}

Barkan, A., Welch, R.C. and Mertz, J.E. (1987) Missense mutations in the VP1 gene of simian virus 40 that compensate for defects caused by deletions in the viral agnogene. J. Virol. 61, 3190-3198.

Boulikas, T. and Hancock, R. (1981) A highly sensitive technique for staining DNA and RNA in polyacrylamide gels using silver. J. Biochem. Biophys. Methods 5, 219-228.

Buonagurio, D.A., Krystal, M., Palese, P., DeBorde, D.C. and Maassab, H.F. (1984) Analysis of an influenza A virus mutant with a deletion in the NS segment. J. Virol. 49, 418-425.

Chanock, R.M., Murphy, B.R., Collins, P.L., Coelingh, K.V.W., Olmsted, R.A., Snyder, M.H., Spriggs, M.K., Prince, G.A., Moss, B., Flores, J., Gorziglia, M. and Kapikian, A.Z. (1988) Live viral vaccines for respiratory and enteric tract diseases. Vaccine 6, 129-133. 
Dildine, S.L. and Semler, B.L. (1989) The deletion of 41 proximal nucleotides reverts a poliovirus mutant containing a temperature-sensitive lesion in the $5^{\prime}$ noncoding region of genomic RNA. J. Virol. 63, $847-862$.

Maassab, H.F. and DeBorde, D.C. (1983) Characterization of an influenza A host range mutant. Virology $130,342-350$.

Meignier, B., Longnecker, R. and Roizman, B. (1988) In vivo behavior of genetically engineered herpes simplex viruses R7017 and R7020: construction and evaluation in rodents. J. Infect. Dis. 158, 602-614.

McCahon, D. and Schild, G.C. (1972) Segregation of antigenic and biological characteristics during influenza virus recombination. J. Gen Virol. 15, 73-77.

Murphy, B.R., Buckler-White, A.J., London, W.T., Harper, J., Tierney, E.L., Miller, N.T., Reck, L.J., Chanock, R.M. and Hinshaw, V.S. (1984) Production and charactcrization of avian-human rcassortant influenza A viruses derived by mating avian and human influenza A viruses. J. Infect. Dis. 150, $841-850$.

Murphy, B.R., Chalhub, E.G., Nusinoff, S.R. and Chanock, R.M. (1972) Temperature-sensitive mutants of influenza virus. Il. Attenuation of $t s$ recombinants for man. J. Infect. Dis. 126, 170-178.

Murphy, B.R., Chanock, R.M., Douglas, R.G., Betts, R.F., Waterman, D.H., Holley, H.P., Hoover, D.L. Suwanagool, S., Nalin, D.R. and Levine, M.M. (1980) Temperature-sensitive mutants of influenza A virus: evaluation of the Alaska/77-ts-1A2 temperature-sensitive recombinant virus in seronegative adult volunteers. Arch. Virol. 65, 169-173.

Murphy, B.R., Markoff, L.J., Hosier, N.T., Massicot, J.G. and Chanock, R.M. (1982a) Production and level of genetic stability of an influenza A virus temperature-sensitive mutant containing two genes with $t s$ mutations. Infect. Immun. 37, 235-242.

Murphy, B.R., D.L. Sly, E.L. Tierney, N.T. Hosier, J.G. Massicot, W.T. London, R.M. Chanock, R.G. Webster and V.S. Hinshaw (1982b) Reassortant virus derived from avian and human influenza A viruses is attenuated and immunogenic in monkeys. Science 218, 1330-1332.

Murphy, B.R., Tierney, E.L., Spring, S.B. and Chanock, R.M. (1976) Temperature sensitive mutants of influenza A virus. XI. Transfer of $t s$ lesions in the Hong Kong/68 $t s-1$ [A] virus to the influenza A/Udorn/72 wild type. J. Infect. Dis. 134, 577-588.

Norton, G.P., Tanaka, T., Tobita, K., Nakada, S., Buonagurio, D.A., Greenspan, D., Krystal, M. and Palese, P. (1987) Infectious influenza A and B virus variants with long carboxyl terminal deletions in the NS1 polypeptides. Virology 156, 204-213.

Parvin, J.D., Young, J.F. and Palese, P. (1983) Nonsense mutations affecting the lengths of the NS1 nonstructural proteins of influenza A virus isolates. Virology 128, 512-517.

Preston, V.G., Darling, A.J. and McDougall, I.M. (1988) The herpes simplex virus type 1 temperaturesensitive mutant ts 1222 has a single base pair deletion in the small subunit of ribonucleotide reductase. Virology $167,458-467$.

Sears, S.D., Clements, M.L., Betts, R.F., Maassab, H.F., Murphy, B.R. and Snyder, M.H. (1988) Comparison of live, attenuated $\mathrm{H} 1 \mathrm{~N} 1$ and $\mathrm{H} 3 \mathrm{~N} 2$ cold-adapted and avian-human influenza $\mathrm{A}$ reassortant viruses and inactivated virus vaccine in adults. J. Infect. Dis. 158, 1209-1219.

Shimizu, K., Mullinix, M.G., Chanock, R.M. and Murphy, B.R. (1983) Temperature-sensitive mutants of influenza A/Udorn/72 (H3N2) virus. III. Genetic analysis of temperature-dependent host range mutants. Virology $124,35-44$.

Snyder, M.H., Betts, R.F., DeBorde, D., Tierney, E.L., Clements, M.L., Herrington, D., Sears, S.D., Dolin, R., Maassab, H.F. and Murphy, B.R. (1988) Four viral genes independently contribute to attenuation of live influenza A/Ann Arbor $/ 6 / 60(\mathrm{H} 2 \mathrm{~N} 2)$ cold adapted reassortant virus vaccines. J. Virol. 62, 488-495.

Snyder, M.H., Buckler-White, A.J., London, W.T., Tierney, E.L. and Murphy, B.R. (1987) The avian influenza virus nucleoprotein gene and a specific constellation of avian and human virus polymerase genes each specify attenuation of avian-human influenza A/Pintail/79 reassortant viruses for monkeys. J. Virol. 61, 2857-2863.

Snyder, M.H., London, W.T., Tierney, E.L., Maassab, H.F. and Murphy, B.R. (1986) Restricted replication of a cold adapted $(\mathrm{ca})$ reassortant influenza virus in the lower respiratory tract of chimpanzees. J. Infect. Dis. 154, 370-371. 
Tenser, R.B. and Dunstan, M.E. (1979) Herpes simplex virus thymidine kinase expression in infection of the trigeminal ganglion. Virology $99,417-422$.

Tian, S.-F., Buckler-White, A.J., London, W.T., Reck, L.J., Chanock, R.M. and Murphy, B.R. (1985) Nucleoprotein and membrane protein genes are associated with restriction of replication of influenza A/Mallard/NY/78 virus and its reassortants in squirrel monkey respiratory tract. J. Virol. 53, $771-775$.

Tolpin, M.D., Massicot, J.G., Mullinix, M.G., Kim, H.W., Parrott, R.H., Chanock, R.M. and Murphy, B.R. (1981) Genetic factors associated with loss of the temperature-sensitive phenotype of the influenza A/Alaska/77-ts-1A2 recombinant during growth in vivo. Virology 112, 505-517.

(Received 1 August 1989; revision received 28 September 1989) 